\title{
DOCUMENTATION
}

\section{La statistique des forces hydrauliques dans le monde}

L'utilisation des forces hydrauliques prend un déreloppement de plus en plus considérable, dans tous les pars, à mesure des progrès de l'industrie. L'augmentation du prix des combustibles et du coùt de leur transport, le risque d'en ètre privé en temps de guerre, pour les pars dont la production est insuffisante, ont conduit la plupart des gouvernements à favoriser par diverses mesures la création d'usines hydro-électriques. C'est ainsi qu'en France, par exemple, on a envisagé la création d'une taxe sur l'énergie électrique distribuée et sur le charbon employé dans l'industrie, taxe dont la produit serait employé à équiper dès chutes d'eau. Un projet de loi, en instance devant le Parlement, propose l'ouverture, pendant vingt ans, d'un compte spécial du Trésor, alimenté : $1^{\circ}$ Par des redevances sur l'énergie livrée aux consommateurs: 20 Par les intérèts et les sommes provenant du remboursement des prèts déjà consentis (1). Une telle disposition favoriserait évidemment la création d'usines hydro-électriques, dont l'intérèt est considérable pour la France, qui importe environ 23 millions de tonnes de charbon par an. Une situation comparable existe en divers autres pays.

Une statistique générale des forces hydrauliques du monde entier a été publiée récemment par le Geological Survey des EtatsUnis; cette statistique ne peut prétendre à une précision réelle, car les données sur lesquelles elle est basée varient suivant les pays, et pour certains elles sont assez approximatives. Dans l'ensemble, cependant, les chiffres du Geological Survey donnent une idée générale de l'importance des forces hydrauliques disponibles et de la proportion qui en est utilisée.

D'après cette statistique, les forces hydrauliques utilisées dans le monde entier atteignaient 23 millions de $\mathrm{CH}$ en 1920, 29 miillons en 1923 et 33 millions à la fin de 1926 .

A cette époque, la puissance utilisée et celle disponible au total dans chaque pays (y compris les chutes exploitées) sont indiquées dans le tableau suivant

\section{AMÉRIQUE DU NORD}

\begin{tabular}{|c|c|c|}
\hline Etats-Unis. & 11.721 & 35.000 \\
\hline Canada.............. & 4.556 & 18.250 \\
\hline Mexique & 300 & 6.000 \\
\hline 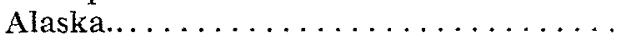 & 43 & 1.000 \\
\hline Terre-Neuve $\ldots \ldots \ldots \ldots \ldots \ldots \ldots \ldots$ & 160 & 400 \\
\hline Costa-Rica. $\ldots \ldots \ldots \ldots \ldots \ldots \ldots \ldots$ & 15 & 1.000 \\
\hline Guatemala......... & 4 & 1.500 \\
\hline Honduras & 3 & 1.000 \\
\hline Nicaragua... . . . . . . & 0,4 & 800 \\
\hline Salvador $\ldots \ldots \ldots \ldots \ldots \ldots \ldots \ldots$ & 2,7 & 200 \\
\hline Panama...$\ldots \ldots \ldots \ldots \ldots \ldots \ldots \ldots$ & 13,3 & 500 \\
\hline Antilles.... & 19,3 & 150 \\
\hline Totaux approximatifs... . & 800 & 66.000 \\
\hline
\end{tabular}

Amérique du Súd

\begin{tabular}{|c|c|c|}
\hline Argentine & 25 & 5.000 \\
\hline 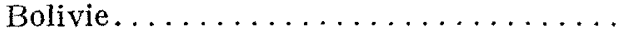 & 13,5 & 2.500 \\
\hline Brésil. $\ldots \ldots \ldots \ldots \ldots \ldots \ldots \ldots$ & 500 & 25.000 \\
\hline Chili. & 114 & 2.500 \\
\hline 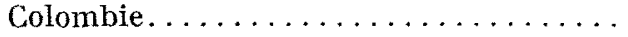 & 25 & 4.000 \\
\hline Equateur.................. & 5,5 & 1.000 \\
\hline 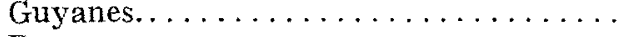 & $"$ & 3.800 \\
\hline Paraguay $\ldots \ldots \ldots \ldots \ldots \ldots \ldots$ & 0,2 & 2.000 \\
\hline Pérou $\ldots \ldots \ldots \ldots \ldots \ldots \ldots \ldots \ldots \ldots \ldots \ldots$ & 55 & 4.500 \\
\hline Uruguay..................... & ") & 300 \\
\hline Vénézuela... & 13 & 3.000 \\
\hline Totaux approximatifs... . . . . . . & 750 & 54.000 \\
\hline
\end{tabular}

(1) Ce projet de loi a été étudié clans le Ciénie Civil da 11 Septembre l!20 tome LXXXIX, no 11 , page 2211 .
Afrigen

Algérie $\ldots \ldots \ldots \ldots \ldots \ldots \ldots \ldots \ldots$

Maroc .........................

Congo belge $\ldots \ldots \ldots \ldots \ldots \ldots \ldots$

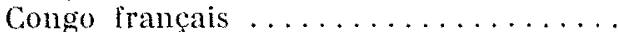

Cameroun français . . . . . . . . . . .

Angola .....................

Nigeria et Cameroun anglais ........

I'nion Sud-Africaine...............

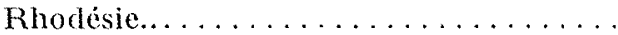

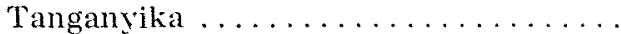

Est africain britannique...........

Abyssinie . . . . . . . . . . . . .

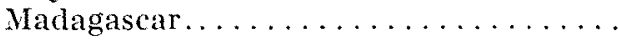

Liberia....................

Est africain portugais.............

Còte d'Ivoire, Dahomey et Togo français.

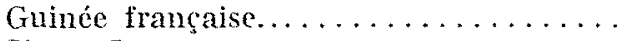

Sierra Leone ...................

Còte d'Or et Togo anglais ..........

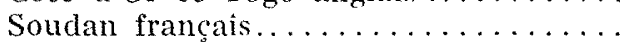

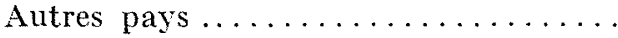

Totaux approximatifs........

\begin{tabular}{cr}
0,13 & 200 \\
$"$ & 300 \\
0,25 & 90.000 \\
$"$ & 35.000 \\
$"$ & 13.000 \\
1 & 4.000 \\
$"$ & 9.000 \\
5 & 1.600 \\
2,5 & 2.500 \\
0,8 & 2.700 \\
9.9 & 4.700 \\
$"$ & 1.000 \\
0,1 & 5.000 \\
$"$ & 4.000 \\
$"$ & 3.700 \\
$"$ & 2.850 \\
$"$ & 2.000 \\
$"$ & 1.700 \\
$"$ & 1.450 \\
$"$ & 1.000 \\
$"$ & 1.000 \\
\hline 14 & 190.000
\end{tabular}

EUROPE

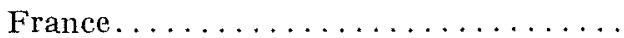

Italie..................

Norvège...................

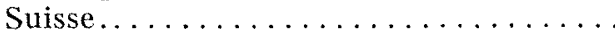

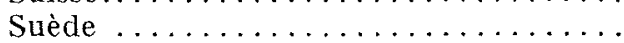

Allemagne..$\ldots \ldots \ldots \ldots \ldots \ldots \ldots$

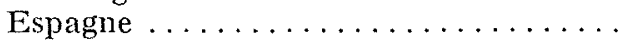

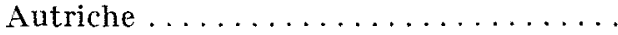

Angleterre . . . . . . . . . . . .

Russie.......................

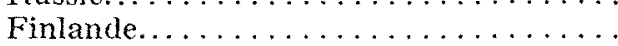

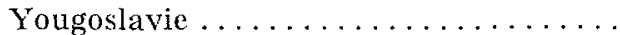

Tchécoslovaquie.................

Pologne.......................

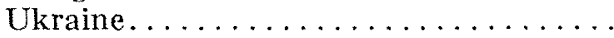

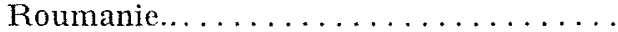

Bulgarie..................

Esthonie......................

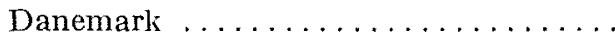

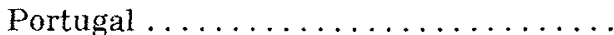

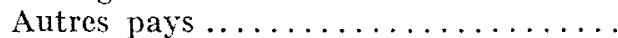

Totaux approximatifs........

2.000

2.300

1.900

1.850

1.350

1.100

1.000

325

250

230

220

180

155

90

40

30

18

17

11

10

$\frac{23}{13.100}$

Asm:

Japon $\ldots \ldots \ldots \ldots \ldots \ldots \ldots \ldots \ldots$

Indes .......................

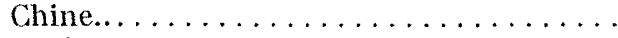

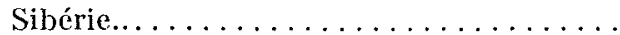

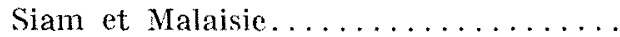

Indochine.....................

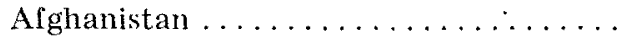

Chosen $\ldots \ldots \ldots \ldots \ldots \ldots \ldots \ldots \ldots \ldots \ldots$

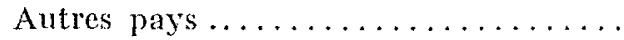

Totaux approximatifs.......

1.750 200

90,8

4,5

2

$18, ;$

$\begin{array}{r}18,3 \\ 0,5 \\ \hline\end{array}$

2.100

5.400

3.800

9.500

2.500

8.000

2.000

1.000

1.660

850

3.000

1.800

3.000

1.000

1. 400

425

16.00

1.200

125

90

300

6.500

$\overline{58.000}$

\section{OcÉante}

Nouvelle-Zélande . ...............

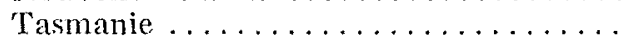

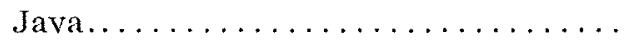
Hawaї. . . . . . . . . . . . . . . . . . 


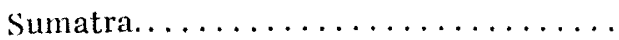

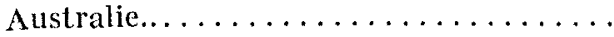

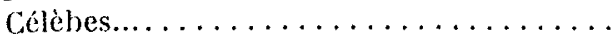

Autres pays $\ldots \ldots \ldots \ldots \ldots \ldots \ldots \ldots$

Totaux approximatifs........

\begin{tabular}{|c|c|}
\hline 20 & 2.000 \\
\hline 2 & 600 \\
\hline 0,5 & 1.000 \\
\hline n & 9.000 \\
\hline 21 & 100 \\
\hline
\end{tabular}

Récapitulation

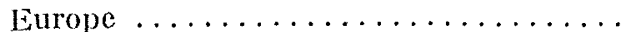

Amérique du Nord $\ldots \ldots \ldots \ldots \ldots \ldots$

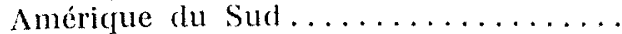

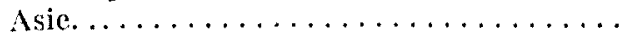

13.100

16.800

750

2.100
58.000

66.000

54.000

69.000

Afrique $\ldots \ldots \ldots \ldots \ldots \ldots \ldots \ldots$

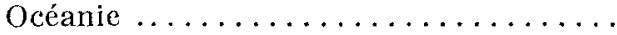

Totaux approximatifs........

$\begin{array}{r}14 \\ 240 \\ \hline 33.000\end{array}$

190.000

17.000

Ces chiffres montrent l'importance considérable de la réserve de force motrice de l'Afrique, et principalement du Congo, où se trouve en puissance le cinquième des forces hydrauliques du monde entier.

Quant aux puissances indiquées pour l'Europe, elles ne semblent pas être données avec toute la précision désirable, puisque la puissance disponible des cours d'eau français n'est évalué qu'à 5.400 .000 chevaux, alors qu'on peut estimer qu'elle est en réalité de l'ordre de 9 millions de chevaux.

Du Génie Civil, tome XLII, No 11, 17 mars 1928.

\section{L'organisation de la production de l'énergie électrique en Grande-Bretagne}

L'importance des richesses de la Cirande-Bretagne en combustibles minéraux a longtemps incité les industriels britanniques i) dépenser sans compter le charbon dont ils avaient besoin. Le gaspillage du combustible est surtout manifeste dans l'industrie de la production de l'énergie électrique, qui compte un nombre ćnorme de petites usines génératrices, mal écjuipées, dont la consommation de charbon par unité d'énergie électrique produite est (le beaucoup supérieure à celle des grandes usines génératrices modernes. Aussi, dès la fin de la guerre, un mouvement se manifeste en vue de réorganiser la production de l'énergie électrique sur des bases nouvelles et, à la suite de longues études, une loi tut promulguée afin d'obtenir le résultat cherché. Cette loi, en late du 23 décembre 1919, et dont le texte a été publié dans ces colonnes (R. G. E., 20 et 27 mars 1920 , p. 411-415 et 445-446.), prévoit l'institution de commissaires chargés de prendre les mesures techniques nécessaires à son application. Quelques résultats ont ité obtenus dans la voie qu'avait tracée le législateur, mais il semble, d'après les informations publiées dernièrement dans la presse techmique britannique. qu'ils soient encore relativement peu importants. C'est également limpression qui se dégage de la note suivante publiée dans le Bulletin Quotidien de la Société d'Etudes et d'Informations économiques, note dans laquelle, après un rappel des origines de la question, sont exposées les décisions des commissaires concernant l'organisation du district qui comprend l'agglomération londonnienne.

L'une des caractéristiques de l'industrie productrice d'électricité en Grande-Bretagne a été jusqu'à ces derniers temps que, s'étant développée sans ordre et sans plan d'ensemble, elle était fragmentée entre un nombre considérable d'entreprises travaillant "en vase clos "sans contact les unes avec les autres, et dont la plupart ne présentaient qu'un caractère purement local.

Une organisation aussi peu rationnelle n'avait que des inconvénients : mauvais rendement du capital, répartition inégale de la production et, pour finir, prix élevé de l'électricité.

Le législateur s'était efforcé d'y remédier par le "Supply Act " de 1919 qui tendait à rationaliser la production et la distribution sur le plan régional, en divisant la Grande-Bretagne en quinze districts et en confiant à une commission, à l'intérieur de chacun d'eux, le soin de réaliser les réformes nécessaires.

Malheureusement, ces commissions n'avaient pas été munies de pouvoirs suffisants : armées pour l'avenir en ce sens qu'elles étaient libres d'établir et de modifier à leur gré les plans d'extension que rendrait nécessaires l'accroissement de la consommation, elles ne l'étaient pas pour liquider le passé, c'est-à-dire briser le monopole dont les entreprises existantes jouissaient en vertu de la loi organique de 1832 , provoquer ou permettre la saine concurrence et réaliser l'interconnexion des établissements producteurs.

Mème lacune en ce qui concerne les prix : libre de fixer les prix maxima, la commission n'avait aucun droit d'intervention dans la fixation des prix minima ni dans l'exploitation.
On conçoit que dans ces conditions, la loi de 1919 ait été un échec : au début de 1926, soit près de sept ans après l'institution du régime, c'est à peine si sept districts avaient été créés sur les quinze prérus.

On connait le défaut du système. Après beaucoup de lenteurs, une nouvelle loi vint $y$ apporter, à la fin de 1916, le correctif nécessaire en rendant obligatoires la coopération et la centralisation, la réduction du nombre des usines génératrices (quitte à indemniser les entreprises lésées ou sacrifiées), la normalisation de la fréquence, la liaison des divers districts, etc..., et en donnant à une commission centrale les pouvoirs nécessaires pour la mise en œuvre de ces réformes.

La commission a déjà déterminé les modifications à introduire conformément au nouveau régime dans le district de l'Ecosse centrale, et les transformations matérielles qui en découlent sont en voie d'accomplissement rapide.

Ses travaux viennent de prendre fin pour un second district. celui de Londres et "Home Counties": ils approchent de leur terme pour ceux des Midland, du Lancashire et du Yorkshire.

Le Times du 18 février expose les modalités de la transformation prévue par la commission pour la région londonienne et les avantages qui en résultent pour les usagers.

Le district comprend onze comtés et empiète sur trois comtés appartenant à d'autres districts; il couvre 8.828 milles carrés; sa population atteint 11 millions d'individus. Sur les 135 usines électriques qui le desservent actuellement, la commission n'en avait retenu d'abord que 13 en tant qu'usines productrices, les autres devenant de simples sous-stations de relai ou étant supprimées purement et simplement. Toutefois, devant l'augmentation régulière de la consommation dans cette région, qui compte parmi les plus peuplées de la Grande-Bretagne, la commission a décidé de porter le nombre des usines principales à 31 ; ces usines seront peu à peu transformées en "supercentrales " de manière à éliminer les autres, mais cette transformation ne s'effectuera que progressivement.

En outre, la commission a décidé de faire construire cuatre grandes usines génératrices.

L'économie réalisée chaque année sur les frais de production de l'énergie grâce à cette organisation plus rationnelle sera de l'ordre de 1 million de livres, pendant les cinc premières années. Mais comme il faudra dépenser plus de 12 millions de livres pour l'établissement du réseau de distribution et d'intercomnexion (6.690.000 livres) et l'agrandissement des usines (5.654.000 livres). le public ne doit pas compter sur une sensible diminution du prix de l'énergie pendant les premiers temps de fonctionnement du nouveau régime. Par la suite, par contre, il est assuré d'y trouver des avantages très appréciables.

$$
\text { R.G.E., 31-3-28. }
$$

\section{Interrupteur blindé pour mines}

Cet appareil présenté à l'Exposition de Cardiff est construit par la Socićté anglaise A. Reyrolles and Co Ltd. Monté sur un pied de fonte qui en permet la fixation sur le sol, cet interrupteur est établi pour $60 \mathrm{~A}, 3.300 \mathrm{v}$., en courant triphasé. La rupture a lieu dans l'huile et les différentes pièces sont étudiécs pour satisfaire aux exigcnecs dics dernières prescriptions anglaises concernant le matériel électrique pour mines.

Engineering, 2 déc. 1927. 


\section{Locomotives et voitures motrices Diesel-électriques}

I.es locomotives et voitures motrices Diesel-électriques semhlent domner la meilleure solution du problème de la traction sur les lignes où l'importance du trafic ne justifie pas l'électrification intégrale, ainsi que sur les lignes secondaires à service fréquent, mais peu chargé. Les avantages de ces véhicules sur les locomotives à vapeur sont les suivants : $a$ ) économie de combustible résultant du meilleur rendement thermique (le rendement total atteint 20 à $25 \%\left(\frac{1}{6}\right)$; b) flexibilité de la transmission et réglage automatique avec la charge, le moteur à combustion interne tournant à sa vitesse optimum ; c) le service assuré par ces locomotives est semblable à relui des locomotives électriques et double de celui d'une locomotive à vapeur : $d$ ) frais d'entretien réduits; e) couple de démarrage et adhérence au démarrage élevés : f) absence d'accouplement mécanique imposant des conditions à respecter dans la disposition des roues motrices, l'empattement, ete. $g$ ) possibilité de la commande à unités multiples. On peut régler la vitesse $\epsilon n$ agissant sur l'excitation de la génératrice accouplée au moteur à combustion interne. la vitesse de ce dernicr étant constante: cette méthode convient surtout aux machines faisant de longs trajets avec une charge sensiblement constante. On peut aussi agir sur lalimentation en combustible du moteur pour faire varier directement sa vitesse, procéclé qut s’applique plutôt aux machines de manœuvre. La génératrice à courant continu qui sert de lien entre le moleur à combustion interne, que l'on doit faire fonctionner au régime de marche économique, et les moteurs de traction aurait pour une puissance constante une caractéristique vitesse qui serait hyperbolique. En praticue pour éviter les tensions élevées qu'une telle caractéristique entraînerait aux faibles courants, on la remplace du côté des faibles courants par un élément de droite plus ou moins incliné sur l'axe des courants. Ce résultat s'obtient en plaçant sur l'excitatrice de la génératrice un enroulement inducteur différentiel parcouru par le courant débité par cette génératrice. Quant au moleur série de traction, lorsqu il est alimenté sous une tension variable comme celle fournie par la génératrice ci-dessus, il a une caractéristique de puissance sensiblement constante. I'auteur termine celte étude par la description de trois types de motrices on locomolives à moteur à combustion interne qui ont d'ailleurs fait chacune l'objet de descriptions détaillées dans différents articles résumés ici-même. Ce sont la motrice du Reading $1 R$. R. Co, lcs motrices des Canadian national Railways et la locomotive Diesel-électrique du professeur Lomonossof. The Electrician, 21 oct. 1927.

\section{Redresseur de courant alternatif à oxyde de cuivre}

Cet appareil, imaginé par L.-O. Grondahl, a déjà fait l'objet d'un article de L.-O. Grondahl et P.-H. Geiger analysé dans "R. G. E. ", $1^{\text {er }}$ octobre 1927 , t. xxir, p. 514. Il est basé sur la curieuse propriété du cuivre oxydé d'avoir une résistance qui varie dans le rapport de 1 à 1000 selon que le courant passe de l'oxyde au métal ou en sens inverse. Il se compose d'une pile de disques de cuivre dont une surface a été oxydée à haute température; le courant qui le traverse est proportionnel à la grandeur des disques et la tension qu'il supporte, au nombre de ces disques. Par exemple, on construit des modèles pour $200 \mathrm{v}$. laissant passer un courant de $10 \mathrm{~m}-\mathrm{A}$. Le rendement d'un tel redresseur est très élevé; il atteint 60 pour 100 , alors que celui des appareils électrolytiques, thermoioniques ou autres est inférieur à 45 pour 100 . L'un des emplois les plus intéressants de cet appareil est la détection et la mesure des courants alternatifs vagabonds qui se rencontrent sur les réseaux de traction. L'utilisation d'un voltmètre à courant continu après le redresseur est en effet beaucoup plus pratique que celle d'un voltmetre à courant alternatif, Ia sensibilité de ce dernier appareil est insuffisante. La Westinghouse Brake and Saxby Signal Company, de Londres, a réalisé un appareil spécialement adapté à cet usage. Elle a également établi des redresseurs pour la charge de batteries d'accumulateurs à une tension qui peut à volonté être de $2,4,6 \mathrm{r}$. I'ar un montage particulier qui comporte plusieurs appareils disposés en pont de Wheatstone et un appareil en dérivation, on peul obtenir non seulement un courant unidirectionnel, mais encore un courant considérablement régularisé plus proche du courant réellement continu que le courant ondulé fourni par les redresseurs d'une façon générale. L'appareil trouve encore une application pour la signalisation des mises à la terre dans les réseaux électriques.
B. E.
Engineering, 11 novembre 1927.

\section{Une nouvelle grande centrale suisse, à Handek}

Cette usine fait partie d'un vaste aménagement du bassin de l'Aar, qui doit comporter, après achèvement, les trois plus grandes centrales de la Suisse, prévues à Handeck, Boden et Innétkirchen.

Celle de Handek - la plus élevée et la plus puissante - est actuellement en voie d'achèvement et comprendra 4 groupes verticaux de chacun 28.000/32.000 KW, 11.000 volts tournant à 500 tours. Les alternateurs sont du type à 2 paliers avec la roue de la turbine montée en porte-à-faux sur l'extrémilé inférieure de l'arbre. La vitesse périphérique normale des rotors est de $72 \mathrm{~m} . / \mathrm{sec}$. La hauteur totale de ces engins, avec excitatrice principale et auxiliaire, atteint 10 mètres. Le poids d'un rotor (roue de turbine exceptée) 65 tonnes; celui d'un alternateur complet 165 tonnes.

Elettrotecnica (Cron.), 25-XI-1927.

\section{Détermination du degré de vide optima (le plus économique) pour les turbines à vapeur}

Le procédé exposé dans cette étude repose sur ce fait que la dépense minimum est représentée par l'intersection de deux courbes, déterminables facilement et représentant : l'une, la dépense (en vapeur) en fonction du degré de vide ; l'autre, la dépense pour le maintien du vide en fonction de ce même degré. Les auteurs examinent du reste, successivement, les éléments intervenant pour la construction de ces deux courbes et qui consistent en variation de la consommation de vapeur; du prix de l'installation génératrice de vapeur; de ceux du condenseur de la turbine et des pompes. Ils terminent enfin, par un exemple concret ayant trait à une turbine de $15.000 \mathrm{KW}$.

$$
\text { Electrical Times, 24-2-31-1928. }
$$

\section{L'électrification partielle du réseau $P$. $O$.}

C'est un devoir envers nos lecteurs en même temps qu'un hommage à la personnalité de leur auteur, que de signaler et rappeler ici, l'important "monument technique " que constitue la longue série d'études et d'articles publiés par M. Pardio dans la $R$. $G$. des $c h$. de fer, et traitant de l'électrification du réseau P.-O., entreprise entre Paris et Brive et déjà réalisée entre Paris et Vierzon.

Les techniciens y trouveront tous les menus détails de cette œuvre formidable depuis les données générales clu progranme d'électrification de la Cie, jusqu'à la description des locomotives en service, en passant, évidemment, par l'équipement des usines el des lignes, que nous ne pouvons que citer, cela va de spi.

R. G. des chem. de ler, nov.-déc. 1925 ; fév., marsavril-août-sept. 1926; janv., mars-avril-juil.août-octob.-nov. 1927 . 


\section{Le chauffage électrique industriel}

L'auteur classe les différents procédés de chauffage par l'électricité qu'il ramène à deux grandes catégories : chauffage par l'arc (pour des températures très élevées et utilisé exclusivement pour les fours de fusion) et chaulfage par résistance. Cette dernière catégorie se suldivise elle-même en chauffage direct par résistance (soit que le courant de chauffage traverse la pièce à chauffer, soit que les courants de chauffage soient développés par induction dans la masse métallicque à chauffer), et chauffage indirect suivant lequel les éléments chauffants élèvent la température d'un four dans lequel sont placécs les pièces à chauffer. C'est le procédé industriel le plus cmployé et celui auquel l'auteur se réfère dans la suite de cette étude. Il expose alors les lois utilisćes clans les études relatives à la chaleur : loi de Joule, à propos de laquelle il donne une courbe montrant la variation de la quantité de chaleur développée dans une résistance en fonction de la variation de tension aux bornes de cette résistance exprimée en pour 100 ; Jois relatives à la transmission de la chaleur. I] établit ainsi l'expressicn de la quantité de chaleur absorbée par un corps passant d'une température $T_{\mathrm{a}}$ à une température $T_{\mathrm{F}}$, et celle de la quantité de chaleur perdue par conduction, par convection et enfin par radiation. Il montre comment, avec la notion de résistance thermique, les problèmes de transmission de la chaleur se traitent d'une façon analogue à ceux de l'électricité et termine en donnant quelques indications sur les valeurs relatives comparées de la propagation par convection $\epsilon t$ par radiation.
G. E. R., oct. 1927 .

\section{Nouveaux progrès de l'électrification des chemins de fer fédéraux suisses}

Le Bulletin des Chemins de fer fédéraux signale, dans son numéro de décembre 1927, que le 15 décembre, la traction électrique a été mise en service sur la section Richterswill-Sargans Buchs, longue de $79,69 \mathrm{~km}$., formant le dernier tronçon de la ligne Zurich-Buchs.

Cette ligne est alimentée par deux sous-stations à ciel ouvert, situées à Sihlbrugg et à Sargans, comportant, la première, quatre transformateurs monophasés, la seconde, trois de ces appareils. Ces transformateurs, d'une puissance de 3.000 KVA chacun abaissent à 15.000 v. la tension du courant qu'elles reçoivent à $60.000 \mathrm{v}$. des usines génératrices.

La traction électrique a été également mise en service le 23 décembre 1927 sur le parcours Yverdon-Bienne-Soleure-Olten, long de $125 \mathrm{~km}$., dernier tronçon de la ligne Genève-Rorschach via Bienne. Le parcours Lausanne-Bienne-Olten, d'une longueur d'environ $160 \mathrm{~km}$., est alimenté en énergie par les trois seules sous- stations de Bussigny, Bienne et Olten. Ces trois sous-stations sont très éloignées les unes des autres, la distance qui sépare les deux sous-stations voisines de Bienne et de Bussigny atteignant $90 \mathrm{~km}$.; néanmoins, elles assurent dans de bonnes conditions, sur une ligne en grande partie à voie unique, un trafic d'environ 500 millions de tonnes-kilomètres.

Ces trois sous-stations, à ciel ouvert, possèdent chacune quatre transformateurs monophasés abaissant la tension de 60.000 à 15.000 v., la puissance de chacun d'eux étant normalement de $3.000 \mathrm{KVA}$ et pouvant atteindre $4.500 \mathrm{KVA}$. Elles sont reliées directement aux grandes sous-stations de Puidoux, Chietres et Rupperswil, situées sur le parcours de la grande ligne de transmission à $132.000 \mathrm{v}$. qui met en communication les usines des Chemins de fer fédéraux du Valais et de la région du Gothard.

$$
\text { R. E. G., 18-11-28. }
$$

\section{Les sous-stations à redresseurs de mercure du Métro et du Nord-Sud de Paris}

On sait que ces deux réseaux sont alimentés en c. c. à $600 \mathrm{v}$. par dix-sept sous-stations de conversion recevant des usines d'Ivry et de Saint-Denis, du triphasé à 10.000 v. Parmi les sousstations, les deux plus récentes (Louvre et Nation) sont équipées avec des redresseurs à vapeur de $\mathrm{Hg}$., logeant dans des espaces plus restreints que les anciens groupes de commutatrices, des puissances bien supérieures. Et, de proche en proche, les autres sont appelées à ètre remplacées par des nouvelles, de ce système; i commencer, tout prochainement, par celles des Lilas et d'Ita- lie : cette dernière allant recevoir celle en service à Nation, laquelle sera, elle-même, remplacée par une nouvelle plus puissante : 3 groupes de $1.200 \mathrm{KWW}$. De même, la sous-station Duhesmes (Nord-Sud) de $6.000 \mathrm{KW}$, va ètre renforcée par deux groupes redresseurs de chacun $1.500 \mathrm{KW}$.

L'étude donne tous détails sur l'équipement de ces sous-stations et les appareils qu'il comporte.

Rev. B. B. C., X-1927.

\section{Ferry-boat à équipement électrique}

Tout récemment la "Southern Pacific "vient de mettre en service, dans la baie de San-Francisco, quatre ferry-boats à équipement électrique. Cet équipement comprend 4 groupes générateurs consistant en moteurs Diesel de $315 \mathrm{~K} W$ à $230 \mathrm{t} . / \mathrm{m}$., accouplés à des dynamos de $275 \mathrm{KW}-250$ v. Directement accouplée est également une dynamo auxiliaire (40 KW-125 v.) pour l'excitation des moteurs et de la génératrice principale ainsi que pour les services auxiliaires du bord, (lesquels peuvent aussi ètre alimentés par un petit groupe spécial, utilisé exclusivement à l'arrèt).

Les moteurs de propulsion (deux par bateau) sont situés aux deux extrémités du navire et reliés en série tant entre eux qu'avec les dynamos. Ils peuvent développer chacun $900 \mathrm{KW}$ à $130 \mathrm{t} . / \mathrm{m}$.

$$
\text { Elettrotecnica (Cron.), 25-XII-1927. }
$$

\section{Les câbles électriques creux}

Les câbles creux s'imposent chaque jour davantage par la nécessité de construire de plus en plus des lignes de très grandes Iongueurs et de puissances élevées jusqu'au maximum compatible avec les limites économiques à ne pas dépasser, comme dépense de matière.

Dans cette intéressante étude, l'auteur passe en revue les princi- paux modes de câbles creux actuellement construits en Allemagne et en Amérique, puis il expose les résultats d'essais déjà obtenus sur l'un d'eux, et termine, enfin, par un exposé, fort honnète, des difficultés de pose que présentent ces nouveaux câbles.

$$
\text { Zeitsch. des vereines Deutsch, 16-7-1927. }
$$

\section{Installation d'un grand turbo-alternateur alimenté par de la vapeur à $35 \mathrm{~kg}$}

Il est encore possible - - certaines circonstances réalisées -.- de demander au charbon (nème dans les pays importateurs) une production d'énergie à des prix de revient comparables - et mème inférieurs - à ceux de l'énergie d' rigine hydraulique. Témoin l'installation des usines Jenny, à Aaran.

Cette installation comporte une chaudière multicellulaire fournissant, à $400^{\circ}$ et sous $35 \mathrm{~kg}$. de pression, 6 tomnes/heure de vapeur destinéc au chauffage et 8 tonnes/heure destinées à l'alter- nateur $(380 \mathrm{KW})$. La turbine est à contre pression; la vapeur d'échappement sert au chauffage, avec - à certains moments adjonction de vapeur vive. Sa vitesse est de $5.000 \mathrm{t} . / \mathrm{m}$.; celle de l'alternateur de $1.500 \mathrm{t} . / \mathrm{m}$.

En finale. l'auteur donne un résumé des dépenses d'installation et d'exploitation faisant ressortir le prix de revient du KWH à 2 centimes 3 (suisses...). 


\section{Une application marquante de la propulsion électrique aux navires}

Cette application se rapporte à un bàtiment marchand de 19.000 tomnes de la "Peninsular Oriental " destiné au Service des Indes (700 passagers) et actuellement en cours de construction.

La vitesse de régime privue est de 19 nouds à l'heure, réalisée par deux groupes turbo-générateurs, comportant des turbines ali- mentées par de la vapeur à $26 \mathrm{~kg}$. 5 . Avec le "Calitornia ", 13.700 tonnes, ce navire sera le plus important exemple d'application de la propulsion électricue aux navires marchands.

Eiletlrolecnica (Cron.), $25 / \times 1 / 1927$.

\section{Application de la loi de similitude aux périodes de formation des tourbillons alternés de Bénard-Karman}

Les éminents auteurs de cette étude ont étudié les périodes de formation des tourbillons alternés qui se produisent dans le sillage de cylindres droits à section circulaire placés dans un champ de secteurs vitesses uniformes : les périodes étant déterminées par lobservation directe, et les vitesses mesurées par chromophotographie. Et après avoir déterminé l'origine et la période d'éta- blissement du régime turbulent ils donnent ici, les résultats de leurs observations, en une profusion de détails (auxquels nous renvoyons nos lecteurs) accompagnés de superbes et intéressantes photos qui illustrent lumineusement la formation des Lourbillons.

C. R. Acas. Sc. Séance du 19-XII-1927.

\section{Le démultiplicateur de fréquence ferromagnétique}

Cette note est l'exposé des observations et des résultats des caiculs effectués par .I. Roubelle sur le phénomène de basse tension, en présence duquel il fut mis par l'étude de la ferrorésonnance sur un circuit composé d un condensateur en série avec une bobine à noyau de $\mathrm{Fe}$ et une résistance.

On sait, par ailleurs, que l'entretien d'une oscillation par un de ses harmoniques supérieurs, a été constaté et observé pour la pre- mière fois, par Fallon et Mauduit, au cours d'essais qu'ils effectuaient sur un interrupteur à haute tension, et que ces éminents précurseurs ont relevé des oscillogrammes correspondant aux divers rapports de démultiplication réalisés, en mème temps qu'ils indiquaient les conditions de résomance -...- anciennes et nouvelles -théoriquement néeessaires tant en charge qu'à vide.

C. R. Acad. Sc. Séance du 19-Xill-1927.

\section{L'installation hydraulique de Balch (Californie)}

Destinée au renforcement de la production de la "San Joaquin Light and Power $\mathrm{C}^{\circ}$ ", cette installation fait partie d'un plan d'aménagement de la Kings River qui permettra d'obtenir de ce fleuve $300.000 \mathrm{KW}$, par exploitation d'une chute de $750 \mathrm{~m}$./s. (la plus haute d'Amérique). La Centrale est prévue pour quatre groupes de chacun $330.000 \mathrm{KVH}$, dont le premier vient d'entrer en service, constitué par un alternateur $33.000 \mathrm{KW}-13.200$ v.-60 pps.-
300 t./m., actionné par deux roues Pelton co-axiales situées de part et d'autre de l'alternateur.

Avant d'arriver à la Centrale, la conduite forcéc, en éléments blindés et soudés, traverse le fleuve enrobée dans un massif du béton reposant sur le lit. L'énergie produite est élevée à $132.000 \mathrm{~V}$. par transfos monophasés, pour son transport aux centres d'utilisation.
Elettrotecnica (Cron.), 25-XI-1927.

\section{INFORMATIONS}

\section{Règles internationales pour les essais de réception des moteurs hydrauliques}

Comme on le sait, la Conférence mondiale de l'Energie a chargé l'organisation internationale déjà existante de la Commission électrotechnique internationale (C.E.I.) d'élaborer des propositions de conventions internationales sur les règles d'essai des installations de force motrice. Pour le clomaine des moteurs hydrauliques, des règles ont déjà pu être arrêtées à la dernière conférence de la (.. F. I. à Bellagio en septembre 1927; ces règles devaient, après avoir reçu l'assentiment des comités nationaux d'Allemagne et de Suède, auxquels, en raison du temps de préparation insuffisant, un droit d'opposition était accordé jusqu'au 31 janvier 1928, être proposées comme règles internationales.

Du côté allemand fut créé d'accord avec le Comité national allemand de la C. E. I, pour l'étude de ces questions, la commission d'hydraulique allemande, qui comprend des représentants des Autorités du Reich et des Etats allemands, ainsi que cles spécialistes ćminents de la science et de l'industrie. A sa première session à Munich, le 4 janvier 1928, cette commission recommanda au comité allemand de la C. E. I. d'adhérer aux résolutions de Bellagio, considérant que celles-ci sont susceptibles d'être modifiées conformément aux progrès de la technique et qu'elles ne doivent être consiclérées que comme un règlement général à l'intérieur duquel il reste à chaque pays une liberté suffisante' pour l'ćtablissement de ses propres règles adaptées à ses besoins particuliers. Sur cette base et en tenant compte de façon approfondie des règles existant en Allemagne et à l'étranger, la commission s'est assigné la tâche supplémentaire de préparer une nouvelle rédaction des Régles allemandes pouvant former la base de propositions pour d'autres conventions internationales.

C'est surtout la question du jaugeage des débits, pour lacquelle sont utilisés toutes les expériences et les résultats de comparaisons disponibles recucillis dans la littérature et fournis par les essais actuellement en cours des laboratoires hydrotechniques allemands et étrangers, qui sera traitée de façon approfondie, de sorte qu'il y a lieu d'espérer que l'on Lrouvera une mesure de comparaison précise. Pour faciliter la compréhension, il sera ajouté au recueil des règles un appendice contenant les définitions essentielles en plusieurs langues. On s'est assuré à cet effet la collaboration d'éminents savants spécialistes de l'étranger. Dans le même but on a décidé de juxtaposer toutes les formules et grandeurs de calcul aussi bien pour le système métrique que pour le système de mesures anglais.

Dans la question de l'évaluation des lorees hydrauliques brutes, on cherchera, en tenant compte des conditions géologiques el météorologiques particulières, à égaliser, d'abord pour les pays européens, les conceptions essentielles, dans le cadre de la C.E.I. 


\section{BIBLIOGRAPHIE}

Les installations téléphoniques automatiques, par F. Lubberger, traduit de l'allemand par $\mathrm{E}$. Munch, avec une préface de E. Reynaud-Bonin.

Cet ouvrage est la traduction de l'édition allemande "Die Fernsprechanlagen mit Wähler-Betrieb (" (Automatische telephonie) dont la bibliographie a déjà paru dans ces colonnes.

Sans revenir sur les mérites de cet ouvrage pour lequel M. Reynaud-Bonin a écrit une intéressante préface, nous ajouterons qu'il convient de féliciter le traducteur, M. E. Munch, de la fidélité avec laquelle il a su rendre les nuances d'un texte portant sur un sujet aussi complexe que la téléphonie automatique.

L. V.

\section{$\star^{\star} \star$}

A la mémoire de Wilfrid-Killian-1862-1925). - Une brochure $16,5 \times 25$ de 106 pages, avec un portrait hors texte.

Cette brochure a été éditée avec le concours des Annales de l'Université de Grenoole et des Papeteries Navarre, par le Comité du souvenir de Charles-Constant Wilfrid-Kilian, membre de l'Institut, professeur à la Faculté des Sciences de l'Université de Grenoble.

Consacrée à la mémoire du regretté géologue Wilfrid-Kilian, cette brochure contient une notice biographique de M. Charles Jacob, la liste chronologique de ses publications scientifiques, les discours prononcés à ses obsèques et à la cérémonie d'inauguration du monument élevé dans la cour de l'Institut de Géologie de la Faculté des Sciences de l'Lniversité de Grenoble (Annexe Très(Cloître), le 8 janvier 1928. La mémoire de Wilfrid-Kilian restera parmi nous, comme celle d'un grand savant, qui fut en outre un beau caractère. Sa vie fût entièrement consacrée à la recherche désintéressée de la vérité.

\section{$\star \star \star$}

(S. V.).

Agenda d'Electricité pour 1928, par MMI. P. Bunet et Ceytre, ingénieurs E.P.C.I. - Ln volume in-18 de 250 pages avec 90 figures. - En vente à la librairie Arthaud-Rey, Grande-Rue, Grenoble. J.-B. Baillière, éditeur, Paris. Prix broché : $15 \mathrm{fr}$.

Que l'électricien soit un homme de science sorti des grandes écoles ou un ouvrier avisé, il a sans cesse besoin de renseignements qui se traduisent par des chiffres dont les colonnes longues et ardues ne peuvent s'enregistrer dans son cerveau. Aussi lui faut-il un aide mémoire qui lui puisse apporter instantanément ce qu'il réclame.

Ce manuel lui est présenté sous une forme commode, celle de l'agenda de poche, contenant des documents nécessaires classés méthodiquement et d'après une documentation rigoureuse.

I.a persomnalité des auteurs est un sùr garant de la bonne conception de cette œuvre nouvelle qui rendra d'excellents services à ceux qui la consulteront. On y trouvera notamment :

Tables des inverses pour les nombres entiers de 1 à 100 . Comparaisons entre les unités de mesures anglaises et celles du système métrique. - Poids des feuilles de différents métaux. Poids des fers carrés, six pans et ronds. - Symboles et signes conventionnels internationaux utilisés en électricité. - Unités électriques et leurs multiples et sous-multiples. - Unités pratiques de travail et de puissance. - Correspondance entre les chevaux et les kilowatts. - Résistances. - Fils en cuivre émaillé. Fils de maillechort. - Courant continu. - Courants alternatifs. Conducteurs en cuivre. - Section en millimètres carrés. - Mesures. - Résistance mécanique des supports de lignes aćriennes sur potelets. - Conducteurs et canalisations. - Fils et câbles en bronze. - Enroulements des dynamos à courant continu. - Sché-

(1) Extrait de la $R . G$. E. et $I . E$. ma d'induits, d'alternateurs et de stators de moteurs asynchrones. - Couplage en parallèle des alternateurs. - Moteurs à courant continu. - Moteurs asynchrones à courant alternatif monophasé, diphasé et triphasé. - Dérangements qui se produisent le plus fréquemment dans le fonctionnement des dynamos alternateurs et moteurs asynchrones. - Transformateurs statiques. - Accumulateurs. - Eclairage et chauffage électriques. - Piles, sonneries, téléphones. - Législation et réglementation. - Conditions techniques auxquelles doivent satisfaire les distributions d'énergie électrique.

\section{$\star^{\star} \star$}

Cours de mécanique à l'usage des élèves des Ecoles nationales d'Arts et Métiers, par L. Guillot. Complément, notions sur la résistance du béton armé, résistance des poutres droites. Un volume in-16 colombier $(16 \times 22)$ de 156 pages avec 94 figures dans le texte broché (225 gr.). Prix net : 24 francs. Frais de port en plus. Béranger, éditeur. En vente à la librairie ReyArnaud à Grenoble.

\section{$\star^{\star} \star$}

Compte rendu du Premier Congrès international tenu à Rome du 21 au 26 septembre 1926. - Lnion internationale des Producteurs et Distributeurs d'Energie Electrique, 26, rue de la Baume, Paris (VIII) $)$ - Un volume 22,5 $\times 29^{\mathrm{c} / \mathrm{m}}$ de 771 pages. - En vente à la Librairie Arthaud-Rey, Grande-Rue, Grenoble.

La création de l'Union Internationale des producteurs et distributeurs d'Energie électrique a été décidée par les représentants des associations professionnelles belge, française et italienne, à la suite du Congrès, tenu à Paris, au mois de juillet 1924 par le Syndicat professionnel français des producteurs et distributeurs d'énergie électrique. Les statuts de la nouvelle Lnion furent mis en vigueur le $1^{\mathrm{er}}$ janvier 1925 et plusieurs groupements appartenant à d'autres nations ne tardèrent pas à venir se joindre aux trois fondateurs. Le premier Congrès international eut lieu à Rome du 21 au 25 septembre 1926 et remporta le plus vif succès. Treize nations y étaient représentées.

Le présent volume donne le compte rendu détaillé des séances de travaik et des diverses communications qui ont été faites.

Il serait trop long d'énumérer toutes ces communications ; nous citerons seulement celles de MM.

Arrighi de Casanova.- Emploi du charbon pulvérisé pour le chauffage des chaudières.

L. Herry. - Emploi des hautes tensions et des hautes températures de vapeur dans les centrales électriques.

Elyio-Salerr. - Les càbles électriques à haute tension.

Couran. - Distillation des combustibles à basse température.

Tochos. - La houille blanche en France.

Boutan. - Les applications de l'Electricité autres que l'éclairage.

qui sont tout à fait remarquables.

Cet ouvrage intéressera tout particulièrement les ingénieurs qui s'occupent de la production et de la distribution de l'énergie électrique, et les élèves des grandes écoles qui voudront se tenir au courant de la science électrique.

$$
\text { (S. Y.) }
$$

\section{$*$}

Modes spéciaux de traction électrique à courant continu, par L. BARBLlion, professeur à l'Université de Grenoble, directeur de l'Institut polytechnique.

Par modes spéciaux de traction électrique, il faut entendre la traction sur route ou sur voie ferrée soit par accumulateurs, soit 
par groupe génerateur a moteur thermique et l'emploi du trolley sur les rihicules routiers.

C'est l'etat actuel des solutions adopties relativement à ces trois questions. que M. Barbillion étudie dans l'ouvrage dont il est question et qui forme un complément au traité sur la traction électrique à courant continu publié antérieurement par l'auteur.

La premiere des quatre parties en lesquelles l'ourrage est divisé, indique les tendances modernes les plus marquées en ce qui concerne la traction électrique.

La deuxième partie concerne létat actuel de la traction électrique par accumulateurs. A ce sujet, l'auteur dome les comptes rendus des essais contrôlés effectués en France sur des véhicules à accumulateurs, de 1923 à 1924 et indique les caractéristiques des autobus de la ville de Lyon.

Dans la troisième partie, l'auteur envisage la traction par trolley des véhicules routiers et après avoir établi une comparaison économique de ces engins avec ceux mus directement par moteur à essence, il décrit l'équipement des lignes d'omnibus à trolley de Mlodane à Lanslebourg et du réseau du Gard.

Enfin, la quatrième partie intitulée "Traction thermo-électrique" contient la description d'un certain nombre de locomotives pourvues d'équipement avec groupe générateur à moteur Diésel. L'extension de ce système aux véhicules routiers et les réalisations accomplies dans cette voie font l'objet des dernières divisions de cette quatrième partie.

L'actualité des sujets traités par M. Barbillion et les nombreux renseignements que contient son ouvrage en rendra la lecture aussi profitable qu'intéressante à toutes les personnes qu'intéressent les questions de traction électrique.

\section{$\star \star \star$}

L. V.

Les machines électriques industrielles, par R. BARDis, ingénieurélectricien E. S. M. E.

Ce petit ouvrage constitue un exposé très élémentaire de la théorie des machines électriques industıielles : génératrices et moteurs à courant continu et à courant alternatif ; transformateurs statiques et rotatifs. Pour chaque type de machine étudiée, l'auteur indique les conditions ci'utilisation, les particularités de montage et les principes d'entretien.

En résumé, ce livre contient, sous une forme concise, les notions essentielles que tout électricien ou usager de machines électriques doit posséder sur ces dernières pour en assurer judicieusement la conduite et l'entretien.

$$
\text { L. V. }
$$

Schémas et règles pratiques de bobinage des machines èlectriques, par F. TonIces, ingénieur-électricien et Adr. Curchod, ingénieur E. S. E.

On reproche parfois aux manuels d'électrotechnique de présenter l'étude des bobinages des machines électriques sous une forme aride et propre à décourager les débutants. Le petit ouvrage de MM. Torices et Curchod échappe à cette critique et le succès remporté par les deux premières éditions montre qu'il répond bien au but que s'étaient proposé ses auteurs, c'est-à-dire de réunir sous une forme aussi simple que possible les éléments nécessaires à l'électricien appelé à s'occuper soit de la construction, soit de la réparation des machines électriques.

Après un rappel des notions d'électricité et de magnétisme, les auteurs développent les considérations générales propres à faciliter la compréhension des phénomènes sur lesquels repose le principe des machines électriques. Vient ensuite la partie essentielle relative aux bobinages. Se basant sur le fait qu'un schéma est plus facile à comprendre qu'un énoncé de règles, si clair soit-il, les auteurs ont donné dans leur ouvrage un grand nombre de schémas de bobinage relatifs aux stators et rotors de moteurs ou de génératrices, et également, aux transformateurs statiques.

L. V.

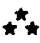

Instructions sur l'exécution des Installations Electriques, établies par le Groupement des Associations françaises des Propriétaires d'Appareils à Vapeur et l'Association des Industriels du Nord. Edition 1924, revue en 1927. Accompagnée de Commentaires rédigés par MM. J. Toupet, ingénieur principal, et V. Kammerer, ingénieur en chef de l'Association Alsacienne, 1 vol. 234 p., 25 fig. - Prix : 15 francs aux sieges des diverses Associations et à la librairie Arthaud-Rey, Grande-Rue, Grenoble.

On sait que les Associations françaises de proprićtaires d'appareils à vapeur ne se bornent pas à la surveillance des appareils à vapeur, mais que depuis longtemps (30 ans pour certaines), elles ont organisé sur les mêmes bases, et avec le même souci d'impartialité et de technicité, des services de contrôle des installations électriques. Un tel contrôle comporte forcément des règles, instructions et conseils, que les Assiociations se sont dès 1900 appliquées à codifier sous la forme d'instructions sur le montage des installations électriques. Après un premier remaniement en 1907 à la suite de la promulgation du décret du 11 juillet de la même annce, les Associations viennent de faire paraitre une $3^{\mathrm{e}}$ édition complètement refondue et mise à jour en tenant compte des progrès les plus récents de la technique et de la grande expérience acquise par elles en matière de sécurité et de bon entretien des installations électriques.

Cet ouvrage constitue un excellent guide et un code complet pour l'exécution des installations électriques de toute sorte, mais surtout des installations industrielles, et il sera des plus utiles non seulement aux installateurs, mais à tous les directeurs d'usine, ingénieurs et chefs d'entretien, ainsi qu'aux élèves ingénieurs de nos grandes Ecoles techniques.

(S. V.)

La Houille Blanche, L'Electrification des Chemins de Fer du Mlidi et l'Essor Economique de la Région du Sud-Est. - Publication de grand luxe de 368 pages. Format $21 \times 27 \mathrm{c} / \mathrm{m}$ avec 300 gravures. En vente à la librairie Arthaud-Rey, Grande-Rue, Grenoble, et chez l'éditeur L,e Sud-Onest Economique, 6 place St-Christoly, Bordeaux. Prix : 22 francs.

Le Sud-Ouest Economique, continuant la série de ses belles monographies régionales, publie un splendide numéro de 260 pages, La Houille Blanche.

Ce numéro étudie plus spécialement l'Electrification des Chemins de fer du Midi, l'Essor économique de la région et le magnifique avenir du Tourisme dans le Sud-Ouest.

Précédé d'une lettre préface de M. André Tardieu, ministre des Travaux Publics et d'une lettre de S. Exc. Quinones de Leon, ambassadeur d'Espagne à Paris, cet ouvrage contient de remarquables études de M. Paul, directeur de la Compagnie des Chemins de fer du Midi et des principaux ingénieurs de cette compagnie, MM. Godard, Bachellery, Villeneuve, Leboucher, Garau, ainsi que des articles de MM. Hubert Lagardelle et Henri Martin, sur l'essor économique de la région du Sud-Ouest, l'électrification rurale et les principales entreprises industrielles.

Une place importante est réservée au Tourisme avec des articles de MM. J. Arnouil, E. Rochelle, Le Bondidier, Pierre Dumas, Comte de Rivière, $D^{r}$ H. Flurin, $D^{r}$ Raymond Molinery, G. Combeléran.

La présentation de l'ouvrage est remarquable. Plus de $300 \mathrm{gra}^{-}$ vures hors texte et dans le texte, aquarelles et dessins de MM. $\mathrm{H}$. d'Hauterive, Ballé-Narcy, photographies de Mme Jacmart, de MM. Bourgeois, Compagnie Aérienne française, Jové, Lumière et Jougla, Mader et Yvon.

Les documents étaient rares sur la Houille blanche dans les Pyrénées, la revue Le Sud-Ouest Economique vient de combler cette lacune, ce superbe travail est un hymne aux efforts faits par la Compagnie des Chemins de fer du Midi pour l'électrification de son réseau.

$$
\text { (S. V.) }
$$

Electrification partielle du réseau de la Compagnie d'Orléans, par M. PARodr, ingénieur-conseil de la Compagnic d'Orléans, préface de M. Paul Dubors, ingénieur des Ponts et Chaussées, directeur honoraire de la Compagnie d'Orléans. - Un volume $21 \times 31 \mathrm{c} / \mathrm{m}$ de 298 pages, 245 figures et VII planches hors texte. Extrait de la "Revue générale des chemins de fer ". - En vente à la librairie Arthaud-Rey, Grande-Rue, Grenoble. Dunod, éditeur.

Comme le dit si bien M. Paul Dubois dans sa préface, l'électrification du Réseau de la Compagnie d'Orléans, bien que partielle, 
est une des plus importantes qui aient été réalisées, non seulement en Europe, mais dans le monde entier, si l'on considère l'ampleur des installations, leur complexité et la diversité des problèmes qui ont été résolus. Il faut louer $M$. Parodi, dont la compétence en matière électrique est aussi universelle qu'incontestée, d'avoir su mettre en évidence, aussi clairement, les principes généraux qui ont dominé la conception et la réalisation d'une ouvre dont il a été le principal artisan.

L'étude de M. Parodi débute par des considérations générales sur la traction électrique, prix de revient, bilan de l'électrification, puis passe en revue les usines génératrices d'Eguzon de coindre, les lignes de transmission d'énergie, les postes de transformation (Chevilly-Chainzy-Eguzon), les sous-stations, les lignes de contact et se termine par des considérations générales sur la constitution des parcs de locomotives et automotrices et la description des différentes sortes de locomotives à grande vitesse et de marchandises en service sur le réseau.

L'électrification partielle des Chemins de Fer d'Orléans, grâce à la compétence avec laquelle elle a été étudiée dans tous ses détails, et aux soins apportés à son exécution, est couronnée d'un plein succès.

(S. V.)

\section{$\star^{\star} \star$}

The measurement of mean spherical candle-power (La mesure de l'intensité lumineuse sphérique moyenne).

Cette brochure, préparée par la division de photométrie du National Laboratory à la demande du Comité des Lampes électriques de la British Engineering Standards Association, a pour but de décrire les méthodes de mesures photométriques effectuées au moyen de la sphère intégrante.

On y trouvera, après quelques indications générales sur l'intérêt de la mesure du flux lumineux total d'une source et sur la théorie de la sphère intégrante, l'exposé des deux méthodes de mesure utilisées dans les laboratoires de photométrie. L'une, dite de substitution, consiste à faire deux mesures successives et dans les mêmes conditions en employant tantôt la source à étalonner, tantôt une source étalon; dans lautre, dite de mesures simultanées, on emploie au contraire, simultanément, les deux sources dans la sphère intégrante.

Cet exposé est suivi de diverses considérations sur l'écran intérieur et sur la fenêtre de la sphère, au sujet de leur forme, de leur position et des erreurs que celles-ci peuvent introduire dans l'exactitude des mesures; des observations se rapportant à la peinture intérieure de la sphère, viennent ensuite.

Les dernières pages sont consacrées à des renseignements pratiques sur la construction des sphères intégrantes et les dispositifs employés pour le changement rapide des sources, la ventilation de l'appareil, la disposition de l'oculaire, etc. Une note concernant les appareils intégrateurs non sphériques termine cette partie dans laquelle le lecteur s'occupant de photométrie rencontrera plus d'un détail utile.

Machinisme et automatisme, par P. MAURER, ingénieur à la Compagnie parisienne de Distribution d'Electricité.

Dans cet aperçu sur l'évolution du machinisme et de l'automatisme, $M$. Maurer se propose d'examiner comment le machinisme peut tendre à l'automatisme et sur quelle base peut être établie la réalisation de cet automatisme. Ce problème considéré dans son ensemble, conduit d'abord l'auteur à développer des considérations relatives à la méthode scientifique en général, dont le machinisme n'est qu'une application. On trouve, dans cette partic de l'ouvrage, l'influence de Henri Poincaré qui a si nettement défini la valeur de la science; et ceci, précisément, à une époque où les progrès de ses applications attiraient l'attention sur elle : une mise au point de son rôle véritable et de son évolution possible s'imposait.

La machine étant définie comme création de l'esprit humain, destinée à être substituée à telle position de l'homme, comment peut-elle tendre à l'automatisme? Ici se présente une difficulté due à la complexité des fonctions que doit remplir le système automatique. M. Maurer procède à une intéressante analyse de ce qu'il appelle l'" automatisation " des machines; il montre d'après ce qui a été réalisé jusqu'à maintenant dans ce domaine, que cette étude a pour base un seul élément, la fonction énergétique: "Pratiquement des fonctions énergétiques convenablement choisies pourront toujours se substituer à la liaison humaine. "

Le point de vue général auquel s'est placé l'auteur et les considérations d'ordre philosophique qu'il a plutôt esquissées que développées donnent à cet ouvrage un intérêt réel qu'apprécieront les lecteurs désireux de se distraire de leurs préoccupations essentiellement techniques.

A. C.

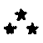

Accumulateurs électriques, par A. CAstex, professeur à l'Institut polytechnique de Grenoble, avec une préface de L. BarbILlion, professeur à l'Université de Grenoble.

I.e champ d'emploi des accumulateurs électriques s'élargit constamment grâce au développement de multiples domaines des applications électrotechniques dans lesquels ces appareils jouent le rôle d'un précieux auxiliaire. A cet égard l'emploi des accumulateurs dans les installations de production et de transformation de l'énergie électrique présente un intérêt tout particulier. C'est pourquoi on ne peut que féliciter M. Castex d'avoir donné dans son ouvrage sur les accumulateurs électriques, une place importante à leurs applications dans les usines génératrices. C'est ainsi que l'auteur, après avoir rappelé dans une première partie les principes du fonctionnement, la théorie et les propriétés des accumulateurs, développe dans la deuxième partie les modes de montage des batteries et les moyens propres à en assurer la charge. Le rôle des survolteurs et des batteries tampons, les moyens propres à en assurer le réglage automatique font notamment l'objet d'une importante et intéressante étude. L'auteur traite ensuite de la conduite et de l'entretien des batteries ainsi que des mesures et essais auxquels elles donnent lieu. Un chapitre est consacré aux batteries transportables pour la traction et la navigation sous-marine. Une étude sur l'accumulateur au fernickel termine cet ouvrage dont la lecture intéressera un grand nombre d'ingénieurs électriciens.

L. V.

Principes de l'électrochimie, par J. Ponsinet, ingénieur des Manufactures de l'Etat.

Cet ouvrage qui fait partie de l'excellente collection Armand Colin, est consacrée à l'exposé des principes et des lois de l'électrochimie.

Dans les deux premiers chapitres, l'auteur étudie le processus de l'électrolyse en se basant sur l'hypothèse de la dissociation en ions. La conductibilité des électrolytes, la mesure de ses facteurs et l'application des mesures de conductivité à l'analyse et à la titrimétrie font l'objet du troisième chapitre. L'auteur aborde ensuite les propriétés des ions : migration, mobilité, hydrolyse, loi de Kohlbrausch. Toutes ces questions sont exposées d'une manière concise, mais néanmoins très substantielle.

Le cinquième chapitre intitulé "La tension électrique dans les phénomènes électrochimiques " a trait à l'étude théorique des piles et de la force contre-électromotrice des systèmes d'électrolyse, d'après les théories de Thomson, d'Helmoltz et de Nernst. L'auteur étudie ensuite, dans le sixième chapitre, la concentration en ions hydrogène qui permet de mesurer le degré d'acidité des solutions, notion d'une importance considérable, en particulier, en chimie biologique. C'est pourquoi ce chapitre a été particulièrement développé dans le but de fournir au lecteur les indications théoriques qui lui faciliteront la lecture des ouvrages spéciaux relatifs à ces mesures.

Dans le dernier chapitre sont esquissées les applications de la théorie des ions électrolytiques, telles que piles, accumulateurs, électrolyse des chlorures alcalins, etc.

L. V.

\section{Annuaire pour l'an 1927 du Bureau des Longitudes.}

Nous avons déjà signalé à nos lecteurs lors de la publication des éditions antérieures de cet annuaire, l'intérêt que présentent les nombreux renseignements qu'il contient. Nous nous bornerons donc à indiquer les adjonctions que comporte la présente édition. 
Dans le deuxieme chapitre, on a introduit les valeurs de la déclinaison magnétique dans l'Afrique occidentale et dans l'Afrique équatoriale. Au troisième chapitre figure le tableau des comètes périodiques dont le retour a été observé et un résume de physique solaire, dù à M. Deslandes.

On a, dans le quatrième chapitre, fait figurer les donnces essentielles contenues dans la notice sur les nouvelles unités légales; enfin, dans le cinquieme chapitre, la mise à jour des tableaux a pu être faite normalement avec des évaluations et des recensements récents.

La liste des villes de 200.000 habitants a été mise à jour avec soin. De plus on a indiqué les chiffres provisoires du recensement de la France, au 6 mars 1926.

I'annuaire renferme en outre deux notices scientifiques. I.'une due à M. Bigourdan, sur les comètes et les élćments d'une statistique cométaire, l'autre de M. A. de la Baume-Pluvinel sur la dernière session de l'Lnion astronomique internationale. tenue à Cambridge du 14 mars au 22 juillet 1925 .

\section{$\because$}

1. Y.

Le nouveau régime des distributions d'énergie électrique (Loi du 27 février 1925), par (h. Blakvoet, docteur en droit.

La loi du 27 février 1925, intitulée "Loi sur les distributions d'électricité " a profondément modifié la situation des distributeurs d'énergie électrique dont le titre est antérieur à sa promulgation, en même temps qu'elle a créé des titres nouveaux d'un caractère spécial. Notre collaborateur M. Ch. Blaevoet, dont nos lecteurs connaissent la compétence en matière de législation industrielle et commerciale, a écrit le présent ouvrage dans le but de faire comprendre la portée de cette loi, les détails du nouveau régime qui vient d'être institué et les droits et obligations des anciens et nouveaux permissionnaires et concessionnaires.

Les quatre parties en lesquelles se divise cet ouvrage, ont respectivement pour titres : Les services publics au sens matériel et le service public au sens absolu; Entreprises de distribution d'énergie organisées en services publics au sens matériel de l'expression; Modification du régime des permissions de voirie; Modification du régime des concessions antérieures.

Au commentaire de l'auteur, sont joints, pour les besoins de la pratique, une codification des diverses lois relatives aux distributions d'énergie, le règlement d'administration publique du 27 septembre 1926 et une table alphabétique des matières.

L. V.

\section{$\star \star \star$}

Traité théorique et pratique des distributions et canalisations
d'électricité, d'eau, de vapeur et de gaz, par E. PACORET.

Les techniciens savent le rôle consiclérable que jouent dans les installations de toutes sortes les systèmes divers de distribution utilisés à ce jour et leur mode de réalisation effectif, représenté par les conduites et canalisations distributrices des fluides ou agents de force motrice.

L'idée fondamentale qui a guidé l'auteur M. E. Pacorct, clont les nombreux ouvrages sont bien connus de nos lecteurs, est une coordination des multiples questions concrètes se rattachant à ce domaine de l'art de l'ingénieur, coordination raisonnée et appuyée sur une longue expérience professionnelle.

Le tome 1 de cet ouvrage constitue un traité abrégé d'électrotechnique appliquée clans lequel l'auteur a insisté particulièrement sur les questions de distributions et de canalisation.

Le tome II est conçu dans le même esprit que le précédent et forme un traité cle physique industrielle dans lequel les questions se rapportant aux canalisations d'eau, de gaz et de vapeur, sont particulièrement développées.

Cet ouvrage a été élaboré de manière à être à la portée des praticiens de l'industrie qui, à côté de l'ingénieur chargé des études, ont à s'occuper journellement des questions relatives à la transmission de l'énergie motrice sous ses diverses formes. Tels sont les architectes, les entrepreneurs, les chefs d'industrie, puis les contrôleurs surveillants, conducteurs de travaux, etc.

Ajoutons que les nombreuses données numériques contenues dans ce traité seront d'une grande utilité dans l'élaboration des projets comportant des canalisations de toutes natures. En outre, cet ouvrage étant d'un niveau assez élémentaire, les particuliers, pourront $y$ trouver un grand nombre de renseignements sur les appareils (fui entretiennent et règlent la vie sociale, tant sur la voie publique que dans les usines, locaux et maisons d'habitation.

I. V.

\section{$\star^{\star} \star$}

L'Electricité, par Lucien PonNcané, directeur au Ministère de l'instruction publique, édition refondue et augmentée par Paul Buner, ingénieur, ancien vice-président de la Société française des Electriciens.

En écrivant l'ouvrage qui portait ce titre, Lucien Poincarć avait pour intention de s'adresser non pas aux spécialistes ou aux débutants, mais bien au public éclairé, qui s’intéresse au progrès des sciences, pour lui présenter un tableau aussi fidèle que possible de l'état actuel de l'électricité. Ainsi que l'indiquent bien les titres des chapitres (Les machines génératrices; Les moteur's; Le transport de l'énergie électrique; Energie chimique et énergié électrique ; L'éclairage électrique, etc.), l'ouvrage était consacré surtout aux applications de l'électricité dont il indiquait le point de départ et le point d'arrivée, en insistant sur les conséquences pratiques d'ordre général que l'ingéniosité des chercheurs a su tirer des principes, Mais la science et l'industrie évoluent rapidement et un tel ouvrage vieillit vite s'il n'est pas tenu au courant des derniers travaux et de récentes réalisations pratiques des nouvelles théries. La disparition de l'auteur nous a valu de voir 2I. Paul Bunet assumer la lourde tàche de la revision et de la refonte de cet ouvrage, tàche dont il s'est acquitté avec succès, malgré les conditions restrictives qu'il s'est imposées de ne point en accroitre le volume et de rester scrupuleusement dans le cadre de louvrage primitif. Pour y arriver, il a fallu compenser de nombreuses adjonctions de certains chapitres par des suppressions ou des compressions de parties que les découvertes récentes avaient démodées ou transformées. Ces conditions font encore que M. Paul Bunet n'a pas pu domner à certains sujets nouveaux la place qu'ils méritaient ; c'est le cas, en particulier, des communications à distance avec ou sans fil, de la production des hautes fréquences, des propriétés des corps isolants. Quoi qu'il en soit, cette nouvelle édition, tout en étant judicieusement modernisée, a su rester dans la note de l'ouvrage initial, et constitue une documentation précieuse, de lecture facile, fort utile à tous ceux qui veulent avoir un aperçu clair des relations entre les découvertes scientifiques sorties des laboratoires et les perfectionnements pratiques dont l'nsemble assure le progrès constant de l'utilisation de l'énergie électrique. - F. P.

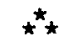

Der phasenverschobene Strom (Le courant réactif), par Richard-F. FALK.

Comment mesurer le courant réactif ou, plus exactement, comment enregistrer l'énergie réactive ? Telle est la question traitée dans la première partie de cet ouvrage. L'auteur expose d'abord le principe des compteurs d'énergie réactive et précise ensuite les énergies, active, réactive et apparente, qui doivent être enregistrées pour permettre l'évaluation du prix de revient; ceci le conduit à établir la théoric du compteur d'énergie apparente, de celui qui totalise l'énergie active et l'énergie réactive (compteur totalisateur qu'il désigne sous le terme abréviatif de "sico ") et enfin, du compteur enregistrant une énergie forméc de deux termes, l'énergie active et une fraction de l'énergie réactive. L'exposé de cette théorie est suivi de quelques indications sur les dispositions à adopter dans le montage des compteurs, notamment dans le cas où deux usines génératrices sont interconnectées.

La deuxième partie traite de la tarification de l'énergie réactive: les principaux modes de tarification, en fonction d'une valeur moyenne du facteur de puissance, en fonction de la puissance perdue, etc., y sont examinés et discutés. Signalons, pour terminer, l'introduction de l'unité kilosin que l'auteur emploie pour l'unité de puissance réactive : le kilosin correspondrait à $1 \mathrm{kv}-\mathrm{A}$ (cette dernière unité étant réservée à la puissance apparente) ou au kilowattré, nom proposé par M. lliovici. --- A. C.

\section{Le Gerant: J. BATAILARD.}

Anciens Etablissements Legendre, 14, rue Bellecordière, Lyon 